\title{
LA SÍLABA EN LA PRODUCCIÓN DEL HABLA DE INDIVIDUOS CON AFASIA DE BROCA
}

\author{
The syllable in production of speech for individuals \\ with Broca's aphasia
}

Hernán Martínez Matos ${ }^{(1)}$

\section{RESUMEN}

Objetivos: analizar los sonidos [+ líquidos] en dos tipos de estructura silábica en la producción de habla de 35 hablantes de español con afasia de Broca con el fin de determinar si dichas estructuras se conservan o se pierden. Métodos: se utilizó una muestra de habla obtenida a partir de conversaciones grabadas con 35 pacientes, 12 hombres y 23 mujeres, con afasia de Broca. A partir de esa muestra se seleccionaron las producciones silábicas de estructura CCV y de estructura CV para determinar las frecuencias respectivas y los posibles errores o sustituciones de fonemas. Resultados: del total de estructuras silábicas analizadas ninguna mostró una estructura diferente a la de la sílaba española. Tanto la estructura CCV como la CV se han mantenido en la muestra de habla afásica analizada. Ninguno de los errores observados contiene secuencias de sonidos mal formadas. Conclusiones: independientemente de los procesos de reducción, asimilación, sustitución, elisión de segmentos, se ha podido determinar que en la producción de habla de los sujetos afásicos hay un gran apego a la estructura silábica. Se sugiere, además, que la sílaba es una unidad funcional que se codifica en el nivel de programación fonética.

DESCRITORES: Afasia de Broca; Medición de la Producción del Habla; Habla

\section{INTRODUCCIÓN}

La sílaba es una unidad fundamental en la mayoría de las teorías fonológicas y una noción bastante antigua. Algunos investigadores ${ }^{1}$ definen la sílaba como la unidad de sonido consistente de una vocal o un pico central de sonoridad y sus consonantes circundantes. Para otros ${ }^{2}$, la sílaba es una "estructura fundamental, básica, de toda agrupación de fonemas en una lengua dada. O dicho de otro modo, la unidad mínima del habla real". Durante las últimas décadas, la noción de la sílaba ha disfrutado de cierta popularidad en la lingüística y la psicolingüística. Su inclusión en las teorías fonológicas le ha permitido a los lingüistas responder varios fenó-

(1) Lingüista; Laboratorio de Fonética; Mestre en Lingüística; Doctorado en Lingüística de la Universidad de Los Andes (Mérida, Venezuela).

Fuentes de apoyo: La investigación que dio origen a este artículo fue apoyada por el Consejo de Desarrollo Científico, Humanístico y Tecnológico de la Universidad de Los Andes (Mérida-Venezuela) a través del Proyecto $\mathrm{H}-940-$ 06-06-Ed y por el Fonacit a través del proyecto ULA-Laboratorio de Fonética-2006000459. menos bastante diversos que serían por otra parte difíciles de explicar ${ }^{3}$. Así mismo, la sílaba ha sido considerada como una unidad de procesamiento en la percepción lingüística. En este ámbito, la sílaba ha sido útil, por ejemplo, para explicar algunos resultados importantes en experimentos que monitorean la audición del habla. Sin embargo, su definición exacta así como su realidad psicológica todavía es polémica. Para algunos investigadores la sílaba es una unidad funcional, para otros es una unidad sustancial; así mientras algunos lingüistas consideran que la sílaba sólo tiene una existencia psicológica y fonológica, otros le atribuyen una existencia fonética, definida por características articulatorias y acústicas ${ }^{2,4}$. Sin embargo, hay dos cuestiones bien admitidas en cuanto a la sílaba: la primera, que la sílaba es una realidad funcional, una unidad de habla real, de la que todo hablante tiene conciencia, incluso desde sus primeros años de vida; la segunda es que la sílaba no sólo es la unidad mínima del habla sino también de la percepción.

La investigación realizada acerca de la sílaba y de su papel en la producción del lenguaje se ha basado en gran medida en el análisis de los errores 
del habla de sujetos sin ningún tipo de patología. Estas investigaciones han sugerido que la sílaba se divide en ataque y rima y que el nivel de la estructura silábica juega un importante papel en el sistema fónico. La estructura silábica del español, particularmente, obedece a reglas de formación específicas ${ }^{5}$ ha elaborado para el español un modelo de estructuración silábica. Según él, la sílaba española tiene dos constituyentes inmediatos: la rima $(R)$ y el ataque (A). La rima es el constituyente obligatorio que presenta el máximo de sonoridad; el ataque, por su parte, es el elemento marginal, opcional, ubicado a la izquierda. Estos constituyentes para que formen sílabas españolas no anómalas deben sujetarse a las siguientes reglas: a) Regla de ataque: constrúyase un árbol cuya ramificación máxima sea binaria y cuyas ramas dominen segmentos [+consonántico] que no sean adyacentes en la escala universal de sonoridad. Esta regla da cuenta de la consonante o grupo de consonantes que pueden aparecer al comienzo de una sílaba española. Por ejemplo, la b de 'be-so'. b) Regla de rima: 1. regla básica: constrúyase un árbol de ramificación máxima binaria, cuya rama izquierda obligatoria domine segmentos [+silábico, -consonántico] y cuya rama derecha opcional domine segmentos [-silábico]. Esta regla da cuenta de las sílabas cuya rima está formada por una vocal ('a-éreo), por una vocal seguida de una glide (diptongo) (por ejemplo, 'hoy'), o por una vocal cerrada por consonante ('mar'). 2. añada un segmento [-consonántico] a una rima. Tal segmento es una glide, quedando de este modo satisfecha la formación de sílabas constituidas por un diptongo creciente ('pie'), o por un triptongo ('buey'). 3. añada el segmento /s/ a la derecha de una rima existente ('austral', por ejemplo).

Particularmente, las combinaciones consonánticas prenucleares formadas por un grupo líquido, es decir, por una obstruyente + una líquida, responden a la regla de ataque. Sólo este tipo de combinación de consonantes puede aparecer al comienzo de morfemas españoles: pr, pl, br, bl, tr, tl, dr, kr, kl, gr, $\mathrm{gl}, \mathrm{fr}, \mathrm{fl}$. Estas reglas están marcadamente establecidas en la intuición del hablante, forman parte de su competencia lingüística.

Ahora bien, examinando el habla de individuos con afasia de Broca se ha podido investigar también el papel y la estructura de la sílaba. En este sentido específicamente, algunos autores ${ }^{7-9}$ han notado que los pacientes afásicos de Broca tienden a eliminar los sonidos líquidos y no los oclusivos en sílabas OLV (Oclusiva, Líquida, Vocal). Este hecho vendría a confirmar la visión de Levelt, según la cual la sílaba se codifica en programas articulatorios. Los estudios sobre los mecanismos articulatorios en la producción del habla han aportado resultados importantes relacionados con los patrones de articulación afásicos. A partir de los datos provenientes de electromiografías realizadas a 6 individuos hablantes de inglés con afasia de Broca algunos investigadores han sostenido que los patrones de coarticulación ${ }^{10}$ y de frecuencia silábica ${ }^{1,3,6,7,11}$ en la producción de afásicos de Broca son similares a la de los hablantes normales. De estos hechos se desprende la idea de que los afásicos de Broca, a veces, son incapaces de programar los articuladores con precisión y otras, seleccionan equívocamente un fonema. Desde esta perspectiva, los afásicos de Broca incurren en errores tanto de ejecución como de planificación. Este hecho también ha sido señalado en otras investigaciones ${ }^{12}$. La mayoría de los trabajos sobre la producción de la sílaba, tanto en español como en otras lenguas, como decía, se han concentrado en establecer las funciones de la sílaba en el procesamiento del lenguaje y sus contextos de aparición y de error en hablantes sanos y no tanto en hablantes con algún tipo de trastorno del lenguaje. En esta investigación se realiza un análisis de dos tipos de estructura silábica contentivas de sonidos [+ líquidos]: (1) en combinación con una obstruyente al inicio y en posición interna de palabra y; (2) en combinación con vocal en inicio y en posición interna de palabra en la producción de habla de 35 hablantes nativos del español con afasia de Broca:

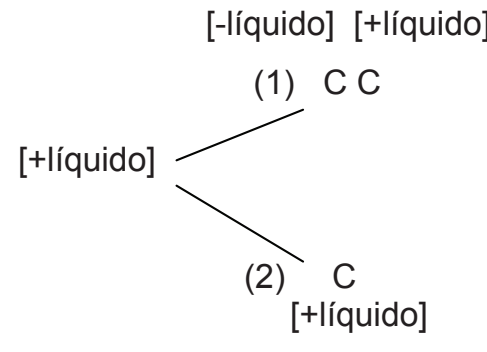

Específicamente, del tipo (1) analizaremos:
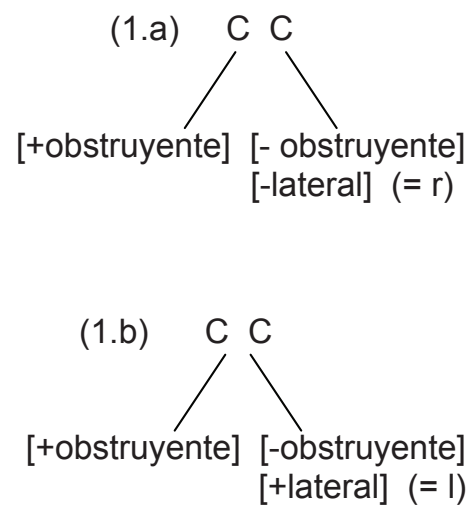

Rev CEFAC, São Paulo, v.10, n.4, 484-489, out-dez, 2008 
Del tipo (2) analizaremos:

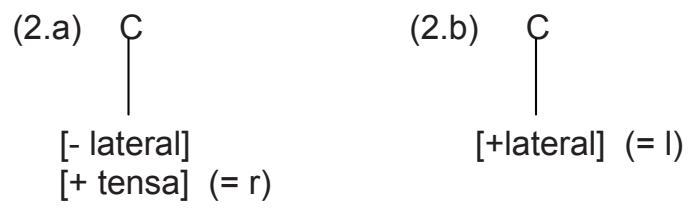

Particularmente, este estudio tiene por objetivos, dando por sentado que la sílaba cumple una función en la producción del habla, (1) mostrar la frecuencia de esos dos tipos de sílabas y (2) determinar si las estructuras silábicas se conservan o se pierden (en cuyo caso nos ocuparíamos de establecer el nivel de procesamiento lingüístico en el que se produce el error) en la producción del habla de los afásicos de Broca pues se considera que estos sujetos pueden presentar problemas específicos en la producción de sílabas que contengan fonemas líquidos.

\section{MÉTODOS}

La muestra de habla afásica que constituye el corpus del presente estudio fue recogida con pacientes del Instituto Autónomo Hospital Universitario de Los Andes (IAHULA), Mérida, Venezuela. Dicha muestra fue obtenida de grabaciones de conversaciones elicitadas a partir de cinco imágenes con situaciones cotidianas diseñadas especialmente contentivas de palabras frecuentes en español con la estructura silábica objeto del estudio: 1.a y 1.b (55 palabras) y 2.a y 2.b (129 palabras) respetando las frecuencias de este tipo de estructura silábica en la lengua española. Las conversaciones se dieron entre dos terapistas del lenguaje y cada uno de los 35 pacientes (12 hombres y 23 mujeres), diagnosticados con afasia de Broca (6 hombres y 12 mujeres con daño cerebral a nivel cortical anterior izquierdo en el lóbulo frontal inferior, producto de tumor cerebral; 4 hombres y 4 mujeres con daño cerebral a nivel cortical y subcortical anterior izquierdo en el lóbulo frontal inferior, producto de un derrame cerebral; 2 hombres y 7 mujeres con daño cerebral a nivel cortical anterior izquierdo en el lóbulo frontal inferior y en el lóbulo parietal inferior, producto de infarto cerebral). Esta clasificación fue hecha por los neurólogos a partir de los exámenes de imagenología (resonancia magnética y tomografías computarizadas) realizados a los pacientes en el IAHULA.

Con respecto al tiempo de lesión de los pacientes:

La edad promedio de los sujetos participantes del estudio es de 62 años y el desvío padrón de edad de los pacientes fue de 6.872. Sólo 19 de ellos reciben actualmente terapia de lenguaje. Todos pre- sentaban trastornos en la articulación de los sonidos del habla al momento de la entrevista.

Las grabaciones se llevaron a cabo en el Laboratorio de Fonética de la Universidad de Los Andes, de forma individual. Se utilizó el programa Sound Forge 9.0 para realizar las grabaciones directamente al computador. Fue utilizado un micrófono profesional cardioide Shrure SM58, dispuesto a una distancia de la boca de unos $12 \mathrm{~cm}$ sobre un pie y en ligero ángulo de aproximadamente $30^{\circ}$ de elevación por la parte posterior, para evitar en lo posible el ruido producido por el aire respiratorio y espiratorio. La señal se recogió en formato .WAV con una frecuencia de muestreo de $44.100 \mathrm{~Hz}$ y una cuantización de 16 bits, para su almacenamiento y posterior análisis.

A partir de las conversaciones recogidas con los 35 pacientes se seleccionaron todas las producciones silábicas de estructura $\operatorname{CCV}$ (1.a y 1.b) y de estructura CV (2.a y 2.b) con el fin de cuantificar las frecuencias respectivas y los posibles errores de articulación (omisión o sustitución) de todos los fonemas consonánticos del español venezolano en la producción de dichas sílabas. En vista de la variación obtenida en las conversaciones, fueron excluidas todas las producciones silábicas que no respondieran a las estructuras objeto del estudio.

Este estudio fue avalado por la Comisión de Ética del Consejo de Desarrollo Científico, Humanístico y Tecnológico (CDCHT) de la Universidad de Los Andes, Mérida con el código H-940-Ed.

Los resultados obtenidos fueron sometidos a tratamiento estadístico. Las medidas de frecuencia de las estructuras silábicas cuantificadas fueron sometidas a análisis estadístico con la prueba $T$ de Student, con un nivel significativo (5\%), y con un análisis de medias significativas.

\section{RESULTADOS}

Los resultados se presentan según la información obtenida a partir del análisis estadístico realizado. En la Tabla 1 se presentan los datos referentes a la frecuencia de producción de los grupos líquidos 1.a y 1.b encontrados en las grabaciones de los 35 sujetos.

De la selección hecha a partir de la muestra de habla de los 35 afásicos de Broca se obtuvieron 1330 producciones de grupos líquidos tipo 1.b (Obstruyente+líquida lateral+vocal). Las sílabas encontradas con esta estructura fueron las siguientes: kl (810); pl (307); bl (192); gl (21) + vocal. Sólo se registraron 51 sustituciones: el fonema sustituto aquí es el líquido vibrante [r] (ej. pratos en lugar de platos). Así mismo, 595 sílabas de estructura 
Tabla 1 - Número de sujetos y el tiempo de la lesión cerebrovascular

\begin{tabular}{ccccc}
\hline Tiempo de lesión & 2 meses & $\mathbf{4}$ meses & $\mathbf{4}$ meses y $\mathbf{3 0}$ días & $\mathbf{5}$ meses \\
\hline $\begin{array}{c}\text { No de sujetos } \\
\text { (2 hombres y } 6 \\
\text { mujeres })\end{array}$ & $\begin{array}{c}7 \text { (1 hombre y } 6 \\
\text { mujeres })\end{array}$ & $\begin{array}{c}10 \text { (2 hombres y } 8 \\
\text { mujeres })\end{array}$ & $\begin{array}{c}10 \text { (7 hombres y } 3 \\
\text { mujeres })\end{array}$ \\
\hline
\end{tabular}

tipo 1.a (Obstruyente+líquida vibrante+vocal) se han encontrado en la producción oral de los 35 hablantes: br (245); pr (234); kr (80); tr (36) + vocal. De estas, sólo en 11 se encontró sustitución de un fonema por otro. En total, 1925 producciones de grupos líquidos tipo 1 se detectaron, lo cual corresponde a la producción total por parte de los 35 hablantes de las 55 palabras tipo 1 (a y b) contenidas en las cinco imágenes utilizadas en la conversación.

Por otra parte, en la Tabla 2 se presentan los datos relacionados con las producciones de las estructuras silábicas tipo 2 realizadas por los 35 sujetos.

Tabla 2 - Frecuencias de producción de grupos líquidos tipo 1

\begin{tabular}{|c|c|c|c|c|c|}
\hline \multicolumn{3}{|c|}{ 1.a } & \multicolumn{3}{|c|}{$1 . b$} \\
\hline \multicolumn{3}{|c|}{ Obs+Líq vib+ v } & \multicolumn{3}{|c|}{ Obs+Liq lat+v } \\
\hline$b r$ & (245) & (6) & $\mathrm{kl}$ & (810) & (14) \\
\hline pr & (234) & (2) & $\mathrm{pl}$ & (307) & (21) \\
\hline $\mathrm{kr}$ & (80) & (2) & bl & (192) & (12) \\
\hline $\operatorname{tr}$ & (36) & (1) & $\mathrm{gl}$ & $(21)$ & (4) \\
\hline \multicolumn{3}{|c|}{$595^{*} \quad 11^{* *}$} & & $30^{*}$ & $1^{\star *}$ \\
\hline
\end{tabular}

*Total de producciones realizadas; ${ }^{* *}$ total de errores

4515 producciones silábicas de estructura tipo 2 (129 palabras) fueron registradas en las 35 grabaciones con los sujetos: 2730 de ellas representan la estructura 2.b (I+vocal), mientras que 1785 producciones representan la estructura 2.a ( $\mathbf{r}+\mathrm{vocal})$. Sólo en 105 de las sílabas del tipo 2 (32 tipo 2.a y 73 tipo 2.b) se produjeron sustituciones de fonemas; por ejemplo: chápido en lugar de rápido. El fonema sustituto en este caso fue $[\mathrm{t}]$ ] y no un elemento líquido como en el caso de las sílabas del tipo 1.

Tabla 3 - Frecuencias de producción de grupos líquidos tipo 2

\begin{tabular}{cc}
\hline 2.a & 2.b \\
\hline Líq vib+v & Liq lat+v \\
\hline $1785^{*} 32^{* *}$ & $2730^{*} 73^{\star *}$ \\
\hline
\end{tabular}

*Total de producciones realizadas; ${ }^{* *}$ total de errores

\section{DISCUSIÓN}

Del $100 \%$ de estructuras silábicas analizadas ninguna mostró una estructura diferente a la de la sílaba española. Tanto la estructura CCV (1) como la CV (2) se han mantenido en la muestra de habla afásica analizada. A diferencia de lo que plantean algunos autores $1,3,6,10,13,14$, no se han encontrado estructuras a las que se les haya añadido, elidido o invertido algún fonema.

En este sentido, los resultados obtenidos en este estudio concuerdan con la idea según la cual en la producción de los individuos afásicos de Broca tanto la estructura como la frecuencia silábicas se encuentran intactas y no muestran modificaciones ${ }^{11}$. Este hecho sugiere la existencia de mecanismos de codificación fonológica intactos operando en el input ${ }^{15}$.

Se pudieron observar, además, tres hechos: 1) en los pocos errores encontrados se suelen preservar el número de las sílabas de la palabra pretendida e, incluso, el contorno acentual de la secuencia original; 2) los monosílabos han sido producidos con mucha fluidez a diferencia de las estructuras de más de dos sílabas. Este hecho confirma la idea de otros investigadores según la cual el mecanismo que planifica las representaciones fonéticas encuentra cada vez más dificultades para cumplir su misión cuando una palabra tiene tres o más sílabas ${ }^{1,3,6,7}$; 3) el número de rasgos distintivos que separan al fonema en el error y en la palabra pretendida suele ser uno, máximo dos. Este hecho permite afirmar que existe un alto grado de similitud entre el fonema sustituto y el sustituido dentro de una sílaba; esto contradice la idea de que la eliminación y/o inserción de sonidos en la palabra o sílaba vienen dadas por el contexto silábico ${ }^{6,7,9}$. Tal como se ha señalado en otros estudios ${ }^{9}$, estas tendencias preferentes de sustitución dentro de las estructuras silábicas parecen reflejar factores articulatorios. Este hallazgo hace pensar que muchos de los errores cometidos por los pacientes con afasia de Broca se deben a perturbaciones producidas en un estadio bastante tardío de la producción de las formas fónicas de las palabras, en el que la articulación está mal especificada en el nivel articulatorio, provocando lugares y modos de articulación del tracto vocal anormales, $y$, en otros, un fonema está especificado en lugar de otro. 
Se puede sugerir que los resultados que se encuentran en este estudio indican que la sílaba es una entidad funcionalmente relevante en la producción del habla en español, pues en los pocos casos de error encontrados se observa que la estructura silábica queda intacta, tal como ha sido demostrado por otros investigadores ${ }^{11}$. La evidencia que se aporta sugiere, además, que se trata de una unidad que se codifica en el nivel de programación fonética, así lo han asegurado otros investigadores ${ }^{4}$. Los datos analizados nos llevan a sugerir que los errores fonéticos son producto de una deficiencia en el nivel fonético del proceso de codificación fonológica, lo cual concuerda con la visión de Levelt y otros investigadores ${ }^{10,11}$.

Lo dicho hasta aquí basta para dejar en claro que la estructura silábica obedece a reglas de formación estricta ${ }^{6}$, y que todo hablante las incorpora en el proceso de adquisición del lenguaje. Así mismo se podría afirmar, a partir de estos resultados que, a pesar de que los fonemas líquidos que conforman las estructuras silábicas analizadas son los últimos en adquirirse durante el desarrollo lingüístico, éstos no se pierden en la afasia de Broca.

\section{CONCLUSIONES}

Partiendo de los objetivos planteados al inicio de esta investigación, se sugiere que, independientemente de los procesos de reducción, asimilación, sustitución, elisión de segmentos, etc., en la afasia de Broca hay un gran apego a la estructura silábica, lo que supone que las reglas de formación silábica siguen operando aún en el caso de que la producción sea un neologismo o una palabra muy alejada del modelo. Según los datos presentados en este estudio la frecuencia de los dos tipos de estructura silábica analizados son altos y significativos. Con respecto a la estructura silábica, se pudo observar que las reglas que rigen el proceso de codificación léxico-fonológico siguen operando estrictamente, esto es, las estructuras silábicas analizadas se conservan en la producción del habla de los afásicos de Broca. Ninguno de los errores observados contiene secuencias de sonidos mal formadas, contrariamente a lo que se planteó hipotéticamente al inicio de este estudio. Los pocos errores encontrados en la muestra de habla afásica analizada podrían entrar dentro de los "niveles normales de error" en la producción del habla de cualquier sujeto normal. Desde este punto de vista, los datos que aquí se presentan llevan a sugerir que la sílaba es una unidad funcional que se codifica en el nivel de programación fonética: la sílaba se codifica en programas articulatorios los cuales son representados en un silabario mental y se recuperan durante la codificación fonética.

Las observaciones sobre la estructuración silábica en la muestra de habla de afasia de Broca analizada bien podrían constituir un aporte a la base de datos sobre la que se ha desarrollado la afasiología lingüística contemporánea.

\begin{abstract}
Purpose: to analyze the [+liquid] phonemes in two types of syllabic structure in production of speech of 35 Venezuelan subjects whit Broca's aphasia, with the purpose of determining if these structures are conserved or lost. Methods: a sample of speech was used. It was obtained from conversations recorded with the subjects, 12 men and 23 women. From that sample, the syllabic productions with CCV and CV structure were selected in order to determine the frequencies and the possible errors or substitutions. Results: from the total of analyzed syllabic structures, all of them showed a similar structure to the Spanish syllable. The CCV and CV structures were maintained. None of the observed errors contained sequences of badly formed sounds. Conclusions: it was possible to determine that in the production of speech of the aphasic subjects there is a great link with the syllabic structure, regardless of the reduction, assimilation, substitution, and elision processes. In addition, it is suggested that the syllable is a functional unit codified in the level of phonetic programming.
\end{abstract}

KEYWORDS: Aphasia, Broca; Speech Production Measurement; Speech 


\section{REFERENCIAS}

1. Wilshire CE, Nespoulous JL. Syllables as units in speech production: data from aphasia. Brain Lang. 2002; 84(3):424-47.

2. Obediente E. Fonética y fonología. Mérida: Consejo de Publicaciones de la Universidad de Los Andes; 1998.

3. Baum SR. Consonant and vowel discrimination by brain-damaged individuals: effects of phonological segmentation. J Neuroling. 2002; 15(6):447-61.

4. Gutiérrez N, Palma A, Santiago J. El papel de la sílaba y de la rima en producción del lenguaje: evidencia desde los errores del habla en español. Psicológica. 2003; 24(1):57-78.

5. Harris J. Syllable structure and stress in Spanish. Cambrigde: MIT Press; 1983.

6. Caplan D. Introducción a la neurolingüística y al estudio de los trastornos del lenguaje. Madrid: Visor; 1992.

7. Laganaro M. Syllable frequency effect in speech production: evidence from aphasia. J Neuroling. 2005; 18(3):221-35.

8. Fink M, Churan J, Wittmann M. Temporal processing and context dependency of phoneme discrimination in patients with aphasia. Brain Lang. 2006; 98(1):1-11.
9. Romani C, Calabrese A. Syllabic constraints in the phonological errors of an aphasic patient. Brain Lang. 1998; 64(1):83-121.

10. Katz WF. Anticipatory coarticulation and aphasia: implications for phonetic theories. J Phonetics. 2000; 28(3):313-34.

11. Stenneken P, Hofmann M, Jacobs M. Patterns of phoneme and syllable frequency in jargon aphasia. Brain Lang. 2005; 95:221-2.

12. Aichert I, Ziegler W. Syllable frequency and syllable structure in apraxia of speech. Brain Lang. 2004; 88(1):148-59.

13. Gordon J. Phonological neighborhood effects in aphasic speech errors: spontaneous and structured contexts. Brain Lang. 2002 ; 82(2):113-45.

14. Alajouanine T, Ombredane A, Durand M. Le syndrome de désintégration phonétique dans l'aphasie. Paris: Mason; 1989.

15. Misiurski C, Blumstein SE, Rissman J, Berman D. The role of lexical competition and acousticphonetic structure in lexical processing: evidence from normal subjects and aphasic patients. Brain Lang. 2005; 93(1):64-78.

16. Pietrosemoli L. Consideraciones lingüísticas sobre la afasia. Entr Cie. 1996; 1:265-78.
RECEBIDO EM: 14/08/2007

ACEITO EM: 15/05/2008

Endereço para correspondência:

Universidad de Los Andes

Facultad de Humanidades y Educación Conjunto

La Liria

Postgrado en Lingüística Ed. D, 2do piso 5101

Mérida - Venezuela

Tel: 0274-2401784 / 0414-1798363

E-mail: hjmart@cantv.net 